\title{
ICI and PAPR enhancement in MIMO-OFDM system using RNS coding
}

\author{
M. I. Youssef, A. E. Emam, M. Abd Elghany \\ Electrical Engineering Department, Faculity of Engineering, Al-Azhar University, Egypt
}

\begin{tabular}{l}
\hline \hline Article Info \\
\hline Article history: \\
Received Aug 18, 2018 \\
Revised Sep 17, 2018 \\
Accepted Oct 1, 2018 \\
\hline Keywords: \\
Bit-error-rate \\
Carrier-to-interferance ratio \\
ICI mitigation technqiues \\
MIMO-OFDM system \\
Residue number system
\end{tabular}

\begin{abstract}
The Inter-Carrier-Interference (ICI) is considered a bottleneck in the utilization of Multiple-Input-Multiple-Output Orthogonal Frequency Division Multiplexing (MIMO-OFDM) systems, due to the sensitivity of the OFDM towards frequency offsets which lead to loss of orthogonality, interference and performance degradation. In this paper Residue Numbers as a coding scheme is impeded in MIMO-OFDM systems, where the ICI levels is measured and evaluated with respect to conventional ICI mitigation techniques implemented in MIMO-OFDM. The Carrier-to-Interference Ratio (CIR), the system Bit-Error-Rate (BER) and the Complementary Cumulative Distribution Function (CCDF) for MIMO-OFDM system with Residue Number System (RNS) coding are analyzed and evaluated. The results had demonstrated a performance of transmission model with and without RNS.
\end{abstract}

Copyright $(2019$ Institute of Advanced Engineering and Science. All rights reserved.

\section{Corresponding Author:}

M. Abd Elghany,

Electrical Engineering Department,

Faculity of Engineering, Al-Azhar University,

Nasr City, Cairo-Egypt.

Email: mohamedgheth@yahoo.com

\section{INTRODUCTION}

In MIMO systems, the information signal is transmitted through the communication link through the usage of various Space-Time Block Coding (STBC) algorithms to achieve either higher transmission data rates or enhanced system BER performance for the same data rate [1], [2]. The OFDM as a multi-carrier modulation scheme had shown its ability to provide high transmission rates, because it has several unique features like robustness to multipath fading overcoming Inter-Symbol-Interference (ISI), high spectral efficiency, immunity to impulse interference, overcoming time dispersion problems, flexibility and easy equalization over wireless communication channels [3], [4].

For MIMO-OFDM communication systems [5], the orthogonality seen in OFDM technique is lost within the sub-carriers due to the sensitivity of OFDM to frequency offset generated from the Doppler shift between the transmitter and the receiver. This results in ICI between the transmitted symbols that cause performance degradation [6].

Different ICI cancellation techniques are currently available like time-domain widowing, pulse shaping and frequency equalization, which reduce the ICI levels and thus improve the BER performance of MIMO-OFDM systems. Still these techniques are costly and high complex either on the transmitter or receiver side. This paper propose an efficient ICI cancellation technique based on the utilization of Residue coding scheme; where the system is analyzed and compared to current mitigation techniques.

In Section 2, the paper provides some basic background on RNS. Section 3 and 4 provide analysis of the ICI and a review for current ICI cancellation techniques respectively. Section 5 describes the proposed MIMO-RNS-OFDM communication system. In Section 6, the simulation results are provided to measure the system performance and finally in Section 7, the conclusion has been provided. 


\section{RESIDUE SYSTEM BACKGROUND}

\subsection{Residue number system review}

The RNS represents large integers by set of smaller ones, and have two features. First, the carry-free arithmetic that enables to perform parallel mathematical operations related to the individual residue symbols. Second, therei is no weight-information between carriers, which prevent error propagation [7].

RNS is defined by selecting $v$ positive pair-wise relative primes $m_{i}(i=1,2,3 \ldots v)$, such that any integer $\mathrm{N}$, describing a message, is represented by the sequence $\left(\mathrm{r}_{1}, \mathrm{r}_{2} . . \mathrm{r}_{v}\right)$ in the range $0<\mathrm{N}<\mathrm{M}_{\mathrm{I}}$ in a unique matter, where;

$$
\mathrm{r}_{\mathrm{i}}=\mathrm{N}\left(\bmod \mathrm{m}_{\mathrm{i}}\right) \text {; The residue digit of } \mathrm{N} \text { upon division by } \mathrm{m}_{\mathrm{i}}
$$

Where; $r_{i}$ is least positive remainder when $\mathrm{N}$ is divided by modulus $\mathrm{m}_{\mathrm{i}}$

$$
\mathrm{M}_{\mathrm{I}}=\prod \mathrm{m}_{\mathrm{i}} \text {; is the information symbols' dynamic range. }
$$

Thus, use the Mixed Radix Conversion (MRC) method [8], to recover symbols. Where for a given set of pair-wise relatively prime moduli $\left\{\mathrm{m}_{1}, \mathrm{~m}_{2}, \ldots, \mathrm{m}_{\mathrm{n}}\right\}$ and a residue state $\left\{\mathrm{r}_{1}, \mathrm{r}_{2}, \ldots . \mathrm{r}_{\mathrm{n}}\right\}$ of a number $\mathrm{X}$, that number can be uniquely represented in mixed-radix form as seen in next:

$$
\mathrm{X}=\left\{\mathrm{Z}_{1}, \mathrm{Z}_{2}, \ldots, \mathrm{Z}_{\mathrm{n}}\right\}
$$

And;

$$
\mathrm{X}=\mathrm{z}_{1}+\mathrm{z}_{2} \mathrm{~m}_{1}+\mathrm{z}_{3} \mathrm{~m}_{2} \mathrm{~m}_{1}+\ldots . .+\mathrm{z}_{\mathrm{n}} \mathrm{m}_{\mathrm{n}-1} \mathrm{~m}_{\mathrm{n}-2} \ldots . \mathrm{m}_{1} ; 0 \leq \mathrm{z}_{\mathrm{i}} \leq \mathrm{r}_{\mathrm{i}}
$$

Where; $z_{i}$ is represented as function of the moduli and residue representations as seen in Table 1 .

Table 1. Representation of $\mathrm{z}_{\mathrm{i}}$

\begin{tabular}{cl}
\hline Parameter & \multicolumn{1}{c}{ Representation } \\
\hline $\mathrm{Z}_{1}$ & $=\mathrm{r}_{1}$ \\
$\mathrm{z}_{2}$ & $=\|\left.\left.\mathrm{m}_{1}^{-1}\right|_{\mathrm{m} 2}\left(\mathrm{r}_{2}-\mathrm{z}_{1}\right)\right|_{\mathrm{m} 2}$ \\
$\mathrm{z}_{3}$ & $=\|\left.\left(\mathrm{m}_{2} \mathrm{~m}_{1}\right)^{-1}\right|_{\mathrm{m} 3}\left(\mathrm{r}_{3}-\left.\left(\mathrm{z}_{2} \mathrm{~m}_{1}+\mathrm{z}_{1}\right)\right|_{\mathrm{m} 3}\right.$ \\
$\mathrm{z}_{\mathrm{n}}$ & $=\|\left.\left.\left(\mathrm{m}_{\mathrm{n} \ldots \ldots . .} \mathrm{m}_{2} \mathrm{~m}_{1}\right)^{-1}\right|_{\mathrm{mn}}\left(\mathrm{r}_{\mathrm{n}}-\mathrm{z}_{\mathrm{n}-1} \mathrm{~m}_{\mathrm{n}-2} \ldots \ldots \mathrm{z}_{2} \mathrm{~m}_{1}+\mathrm{z}_{1}\right)\right|_{\mathrm{mn}}$ \\
\hline
\end{tabular}

\subsection{Redundant residue number system}

The RNS moduli utilized for error detection and correction through implementation of additional RNS moduli as redundancy symbols; that is called Redundant Residue Number System (RRNS). In this configuration, each redundant moduli selected to be greater than any of the other chosen moduli set and don't play any role in determining the system dynamic range. So, an RRNS is obtained by appending an additional $(\mathrm{u}-\mathrm{v})$ number of moduli $\mathrm{m}_{\mathrm{v}+1} ; \mathrm{m}_{\mathrm{v}+2} ; \ldots . . ; \mathrm{m}_{\mathrm{u}}$, where $\mathrm{m}_{\mathrm{v}+\mathrm{j}} \geq \max \left\{\mathrm{m}_{1} ; \mathrm{m}_{2} ; \ldots \ldots ; \mathrm{m}_{\mathrm{v}}\right\}$ is referred to as a redundant modulus, to the previously introduced RNS, in order to form an RRNS of u positive, pairwise relative prime moduli. [9, 10]. For the correction of the error, using the MRC method, a test on each of the information moduli with the two redundant moduli is performed. Through the test it is able to identify and correct the bit which generated the error [11].

\section{ANALYSIS OF INTER-CARRIER-INTERFERENCE}

In MIMO-OFDM systems, the loss of orthogonally between subcarriers, increases the ICI between sub-carriers and degrades the system performance. This is attributed to the Doppler shift generated from sensitivity of the relative motion between both sides of the communication link that caused a frequency offset between sub-carriers, and would result in a reduced signal amplitude and ICI as presented in Figure 1. 


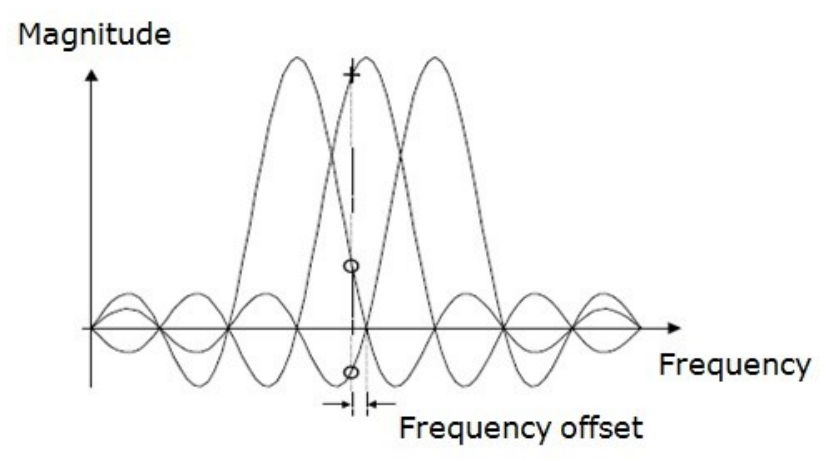

$\mathrm{ICl}$ due to frequency offset

Figure 1. Effect of carrier frequency offset

The frequency offset $(\varepsilon)$ is modeled as shown in Figure 2 where the received signal represented as;

$$
Y(n)=x(n) e^{\frac{j 2 n \varepsilon}{N}}+W(n)
$$

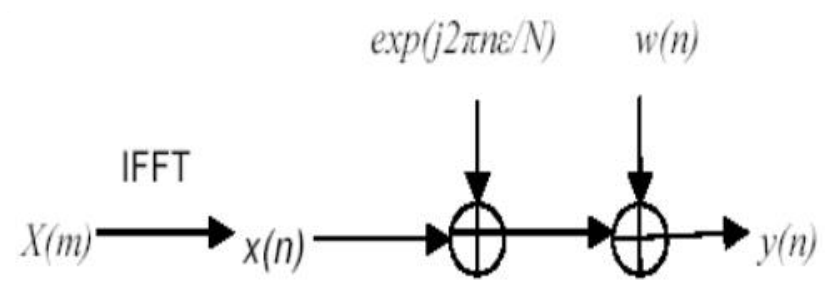

Figure 2. Frequecny offset model

The effect of this offset on the received stream is shown in the received symbol Y(k);

$$
\mathrm{Y}(\mathrm{k})=\mathrm{X}(\mathrm{k}) \mathrm{S}(0)+\sum_{\mathrm{l}=0, \mathrm{l} \neq \mathrm{k}}^{\mathrm{N}-1} \mathrm{X}(\mathrm{l}) \mathrm{S}(\mathrm{l}-\mathrm{k})+\mathrm{n}_{\mathrm{k}}
$$

Where:

$\mathrm{X}(\mathrm{k})$ : Transmitted symbol for kth sub-carrier.

$\mathrm{n}_{\mathrm{k}}$ : The FFT of $w(\mathrm{n})$.

$\mathrm{N}, \mathrm{S}(1-\mathrm{k})$ : Total number of sub-carriers, and ICI components for received signal respectively.

The ICI components are the interfering signals transmitted on sub-carriers, where their complex coefficients are given by;

$$
\mathrm{S}(1-\mathrm{k})=\frac{\sin (\pi(1+\varepsilon-\mathrm{k})}{\mathrm{N} \sin \left(\frac{\pi(1+\varepsilon-\mathrm{k})}{\mathrm{N}}\right)} \exp \left(\mathrm{j} \pi\left(1-\frac{1}{\mathrm{~N}}\right)(\mathrm{l}+\varepsilon-\mathrm{k})\right.
$$

\section{ICI MITIGATION TECHNIQUES}

The accurate frequency and time synchronization are fundamental for OFDM approach. The sensitivity towards generated frequency offset factors leads to loss of orthogonality among carriers and yields in causing inter-carrier interference (ICI), which degrades system efficiency.

The researchers [12]-[14] have proposed numerous ICI mitigation techniques to resolve this problem as; frequency-domain equalization, time-windowing, self-cancellation, and Pulse shaping techniques. These techniques are employed as well for the reduction of the Peak-Average-Power Ratio (PAPR) through the reduction of side lobes in each carrier, and making improvements to the overall signal to noise ratio (SNR) at the receiver. A detailed description of existing ICI mitigation techniques, provided next. 


\subsection{Self-cancellation technique}

The input symbols are modulated to a group of subcarriers with pre-defined coefficients such that the ICI signals would cancel each other in the group. So, one data symbol is modulated into two consecutive sub-carriers, such that the data symbol ' $a$ ' is modulated in the first sub-carrier, and '-a' is modulated in to the second subcarrier. Consequently, the generated ICI between the two sub-carriers will be cancelled.

Through this scheme, it is possible to achieve an improvement in Carrier-Interference-Ratio (CIR) of about $20 \mathrm{~dB}$ for $0<\varepsilon<0.5$, due to the reduction in the ICI levels compared to the standard OFDM system [15]. Furthermore, this technique doesn't need an estimation feedback and is simple in implementation, but on the other hand, due to the redundancy introduced, it is required a larger bandwidth.

\subsection{Frequency domain equalization}

A frequency pilot symbol is inserted between two sub-blocks as seen in Figure 3 where it is able to determine the coefficients of the equalizers that are used in frequency domain [16]. This technique is similar to the Maximum Likelihood (ML) estimation and the Extended Kalman Filter (EKF), which estimate the offset and correct it at the receiver side.

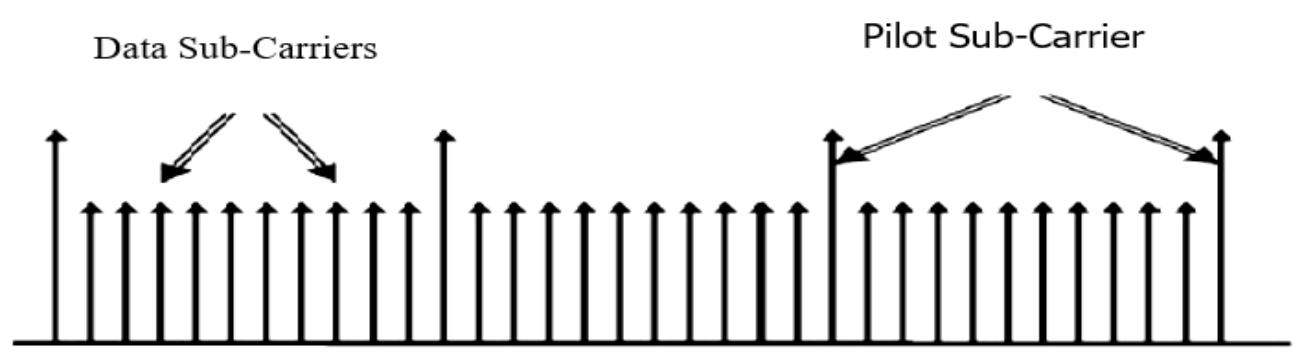

Figure 3. Pilot sub-carrier arrangement

\subsection{Windowing technique}

It is system equalization in time-domain [17], where the transmitted signal is multiplied by an exponential function before calculating its Fourier transform, as seen in (8), to reduce the effect of discontinuities at both ends of the discrete signal.

$$
b_{k}=a_{k}(1-\exp (j 2 \pi n / N))
$$

Where; $b_{\mathrm{k}}$ is the transmitted data samples on the kth subcarrier

This mitigation technique reduces the start and ends of waveform, as well as transients and thus reduces the spectral spreading. Also, it is utilized to decrease the sensitivity towards frequency errors and so reducing BER of the system. All the windows include Hanning, Nyquist, and Kaiser etc, give some reduction in the sensitivity to frequency offset.

\subsection{Pulse Shaping Technique}

The peak power is associated with main lobe of the signal, whereas the ICI power is associated with side lobes. So the objective is to reduce side-lobes amplituide and increase the main lobe. This is done through using a new pulse shaping functions to decrease the side lops in each carrier and consequently, reduce ICI [18].

This technique is very similar to the windowing technique, and even is implemented in similar ways, but their purposes are different. The pulse shaping means choosing a pulse with the desired spectral and orthogonality properties for ICI power reduction.

Several pulse shaping functions are present to perform the requirement as: Raised Cosine pulse (RC), and Square Root Raised Cosine pulse (SRRQ), which presented in (9) and (10) respectively:

$$
\mathrm{P}_{\mathrm{RC}}(\mathrm{f})=\operatorname{sinc}(f \mathrm{t}) \frac{\cos (\pi(\pi \mathrm{ft})}{1-(2 \alpha \mathrm{ft})^{2}}
$$


Where;

$\alpha$ : The roll off factor,

$f$, $t$ : The frequency, and $\mathrm{t}$, respectively

$$
\operatorname{P}_{\text {SRRC }}(f)=\operatorname{sinc}(f \mathrm{t})\left(\frac{4 \alpha}{\pi-(\mathrm{f})^{0.5}} \cos (1+\alpha)\left(\frac{\pi \mathrm{f}}{\mathrm{t}}\right)\right)+\left(\frac{\frac{\mathrm{t}}{4 \alpha \mathrm{f}} \sin (1-\alpha) \frac{\pi \alpha}{\mathrm{t}}}{1-(4 \alpha \mathrm{f} / \mathrm{t})^{0.5}}\right)
$$

Through this technique the side loop power is decreased to reduce the ICI between the adjacent carriers and achieve better bandwidth efficiency, which could be further enhanced through increasing the number of filter coefficients, as, indicated in previous literature [19].

\section{SYSTEM MODEL}

The proposed MIMO-RNS-OFDM system is shown in Figure 4 is initialized with a binary data random source, converted to residue system. The packet is then modulated, coded through the STBC encoder, passed to a Serial-To-Parallel (S/P) converter for parallel transmission, and then passed through an IFFT block and finally transmitted through the antenna. At the receiver side the communication blocks are the reverse of the transmitter.

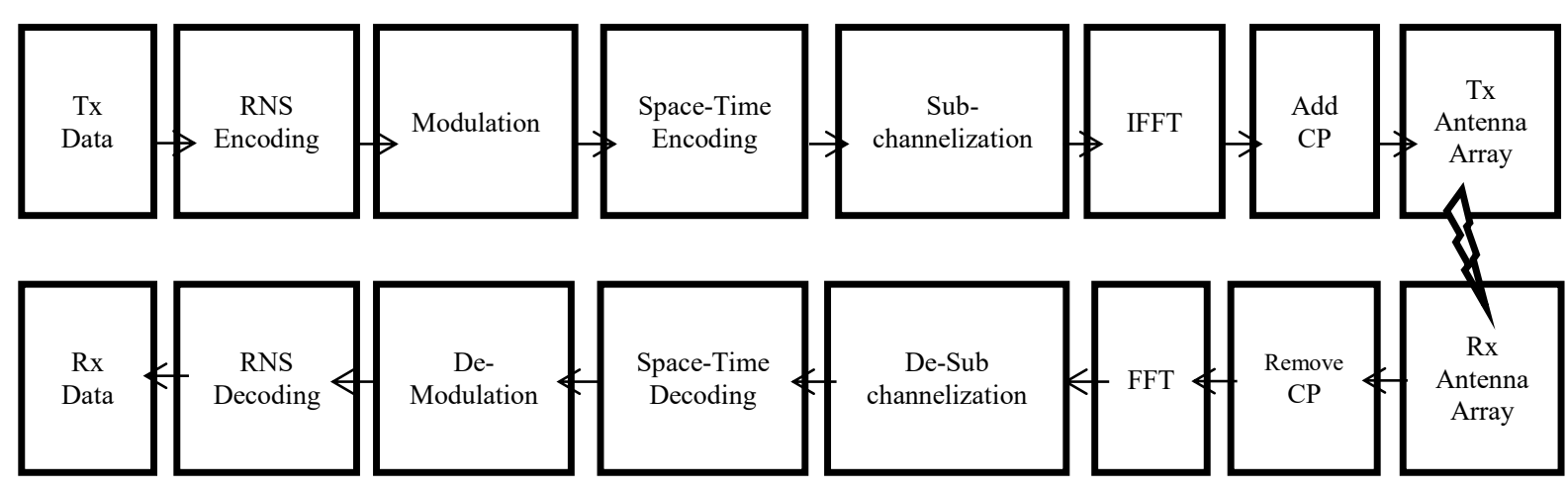

Figure 4. MIMO-OFDM system model

The above system shown in Figure 4 is evaluated by measuring the Carrier-Interference-Ratio (CIR) given in (11), and the Bit Error Rate (BER) of the signal shown in (12), respectively.

$$
\operatorname{CIR}=\frac{|S(k)|^{2}}{\sum_{l=0, l \neq k}^{N-1}|S(l-k)|^{2}}
$$

Where;

$\mathrm{S}(\mathrm{l-k})$ Complex coefficient for ICI components in the receiving signal.

And; the probability of error for M-PSK modulated transmission is given by:

$$
\begin{aligned}
& \mathrm{P}_{\mathrm{ERR}}=\gamma \sum_{k=1}^{\min \left(2,\left[\frac{M}{4}\right]\right)} Q\left(\sqrt{2 \sigma x} \sin \left(\frac{(2 k-1) \pi}{M}\right)\right) \\
& \gamma=\frac{2}{\max (\log 2 M, 2)}
\end{aligned}
$$

Where;

$\mathrm{M}$ is the constellation size

$\rho$ is the SNR per symbol

$\mathrm{x}$ is a chi-square distributed random variable 


\section{SIMULATION RESULTS}

The results obtained from the MATLAB simulations are discussed, where various analysis had been performed on MIMO-RNS-OFDM system to measure its resilience towards ICI. In this simulation, 1000 symbols are 512-QAM modulated and transmitted over a MIMO-OFDM communication system using RNS coding technique with redundant moduli's $(17,13,11,7,5,3)$, where $(11,7,5,3)$ are the information moduli's and the set $(13,17)$ are the redundant moduli's.

\subsection{BER vs. SNR for various offset values for MIMO-RNS-OFDM system}

In Figure 5 the performance of communication system in the presence of varies frequency offset values between the transmitter and the receiver is evaluated and discussed. From Figure 5 it is shown that the system performance is degraded with the increase in the frequency offset, so for small offsets the system has lower BER (better).

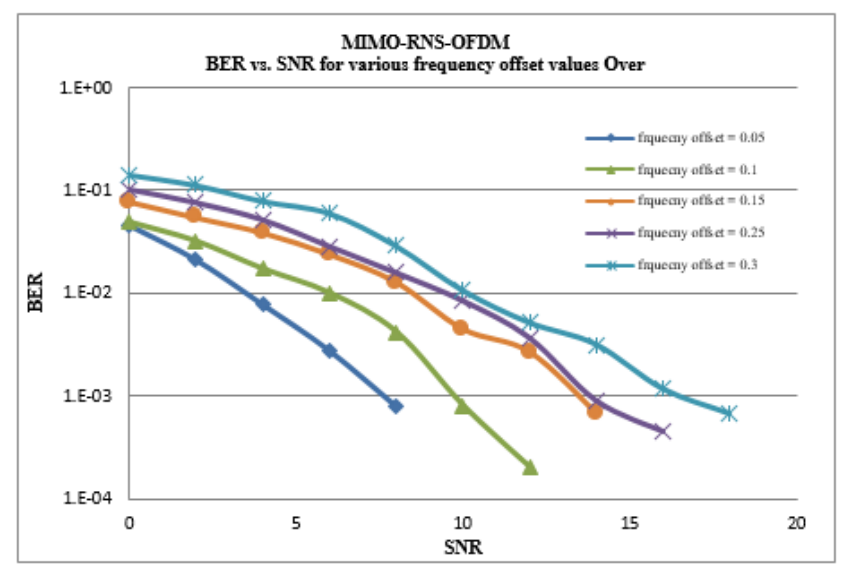

Figure 5. Effect of frequency offset on system performance

\subsection{ICI measurements for MIMO-RNS-OFDM system}

For a pre-defined SNR value (80), the transmission signal error is plotted versus the frequency offset as seen in Figure 6 for OFDM system with and without RNS moduli's $(13,11,7,5,3)$ as coding scheme. Where; it is seen in Figure 6 an absolute $25 \mathrm{~dB}$ improvement when using the RNS scheme, which is better than the achieved improvement using ICI cancelation scheme indicated in Section (4.1). In addition, it is seen that-as expected-as the frequency offset increase this would increase the error due to the increasingly loss of orthogonality between inter-carriers. The RNS coding performance is even enhanced with low offset values due to the inherent properties of RNS that doesn't allow the transmission of error between different moduli's.

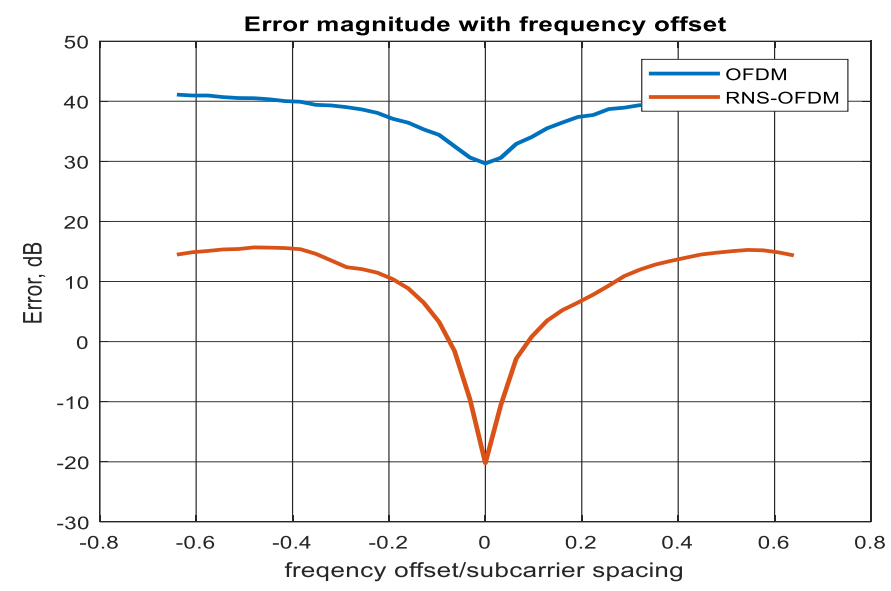

Figure 6. Error for MIMO-RNS-OFDM system 


\subsection{ICI measurements for MIMO-RRNS-OFDM system}

Using RNS technique with redundant moduli's $(17,13,11,7,5,3)$, where $(11,7,5,3)$ are the information moduli's and the set $(13,17)$ are the redundant moduli's, and measuring ICI for the system comparing its value with the communication system without any redundant modus as seen in Figure 7.

Here in Figure 7 the improvement is more than $30 \mathrm{~dB}$, which is better than ICI cancellation scheme and RNS coding scheme seen in Sections (4.1) and (6.2) respectively. Moreover, the system exhibit similar performance as that shown when using RNS as a coding scheme only, as seen in Section (6.2).

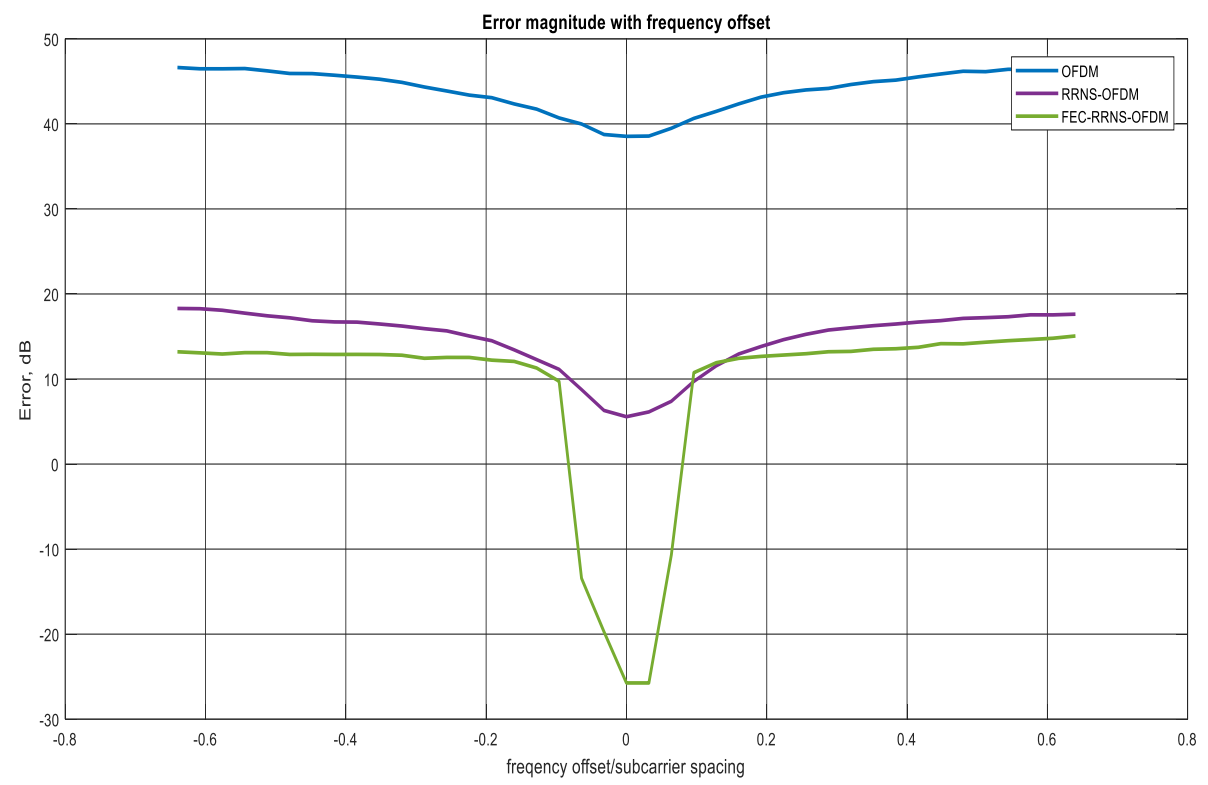

Figure 7. Error for MIMO-RRNS-OFDM systems

\subsection{Effect of RNS moduli selection on ICI performance}

Increasing the order of RNS moduli set and measures the system performance to see the effect of the selection of the RNS on ICI reduction. ICI vs. RNS moduli set shown in Figure 8 it is noted that each time the amplitude of the RNS set increased this would increase the ICI error, and thus the increased signal amplitude would increase directly the interference between adjacent sub-carriers. Now, in the comings subSections (6.5) to (6.8) various mitigation schemes are implemented and analyzed in the MIMO-RNS-OFDM communication system to study and evaluate its performance in combination with Residue coding technique.

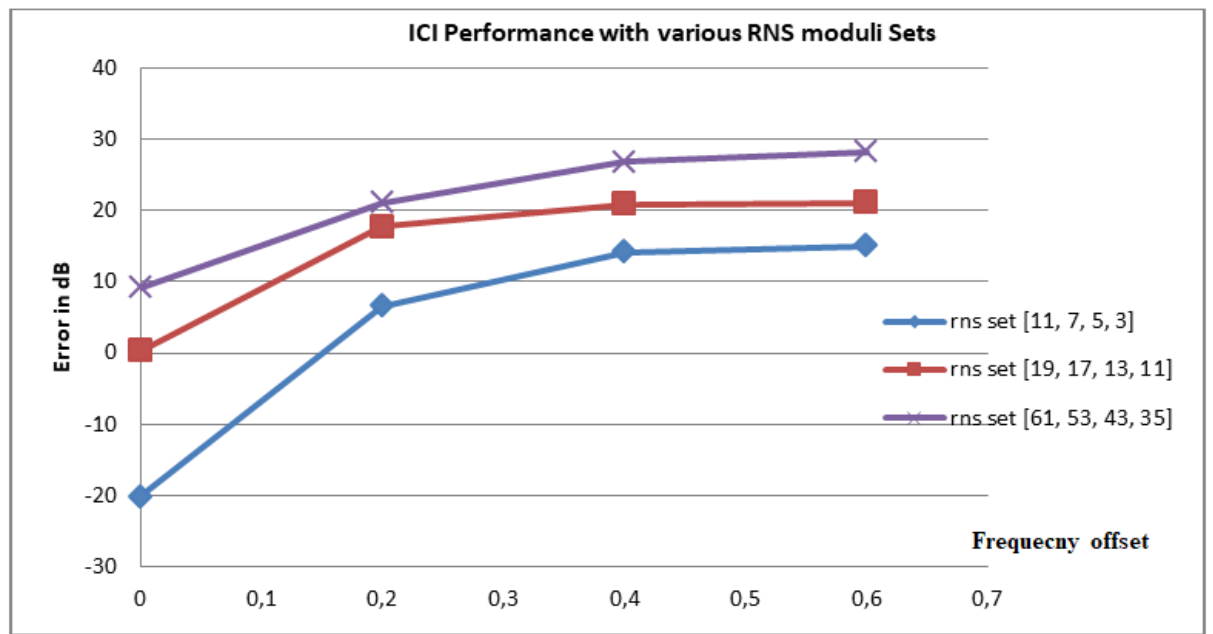

Figure 8. ICI vs. RNS moduli set 


\subsection{MIMO-RNS-OFDM with "frequency domain equalization" scheme}

A frequency domain equalizer is used in the receiver, and the system performance is evaluated as seen in Figure 9 over a Rayleigh fading channel. Using an MLSE equalizer for 32-QAM over a Rayleigh channel@ $@$ SNR=10 dB, the BER for the communication system with error correction is $\sim 2 * 10^{-3}$ while it reaches $6^{*} 10^{-3}$ for the system without error correction. The BER @ $10^{-3}$ the SNR is reduced by $\sim 5.5 \mathrm{~dB}$ when using Equalization mitigation scheme.

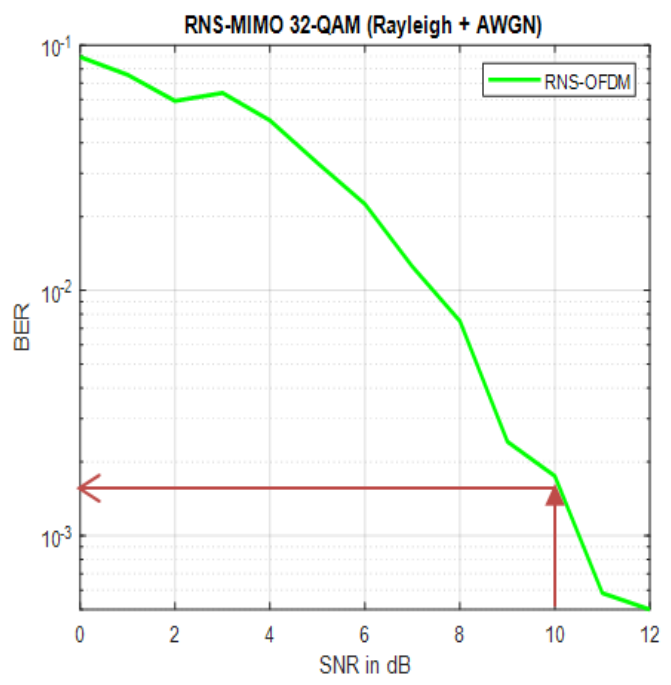

(a) With equalization

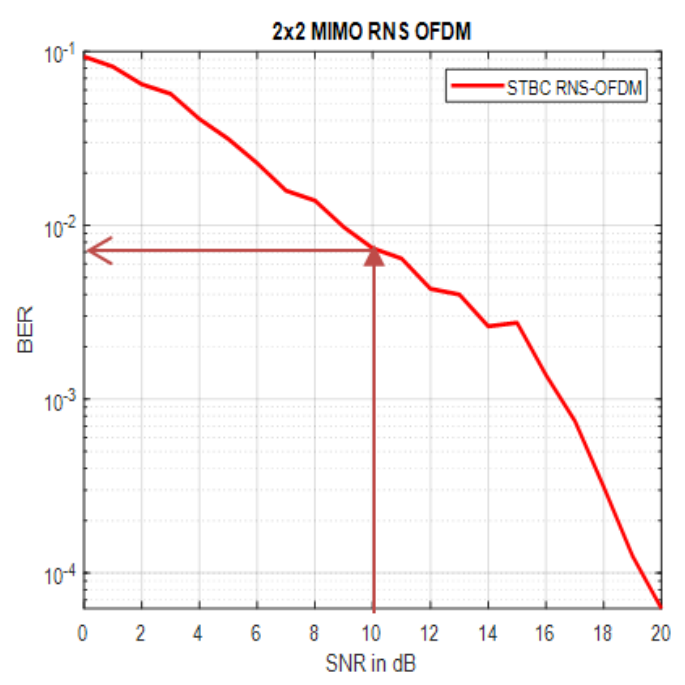

(b) Without equalization

Figure 9. MIMO-OFDM RNS system with/without equalizer

\subsection{MIMO-RNS-OFDM with "self-cancellation" scheme}

Using data conjugate technique in self-cancellation scheme, where the system performance is evaluated as seen in Figure 10 over a Rayleigh fading channel. For a 32-QAM over a Rayleigh channel, using a data conjugate self-cancellation system @ SNR =10 dB, the BER for the communication system with error correction is $3 * 10^{-3}$, while it reaches $7 * 10^{-3}$ for the system without error correction. At BER @ 10 ${ }^{-3}$ the SNR is reduced by around $3 \mathrm{~dB}$ when using Self-cancellation mitigation scheme.

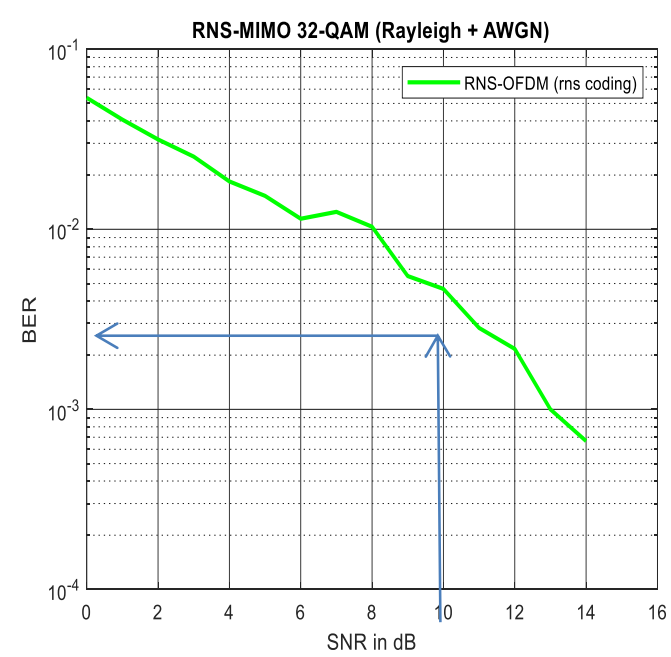

(a) With mitigation scheme

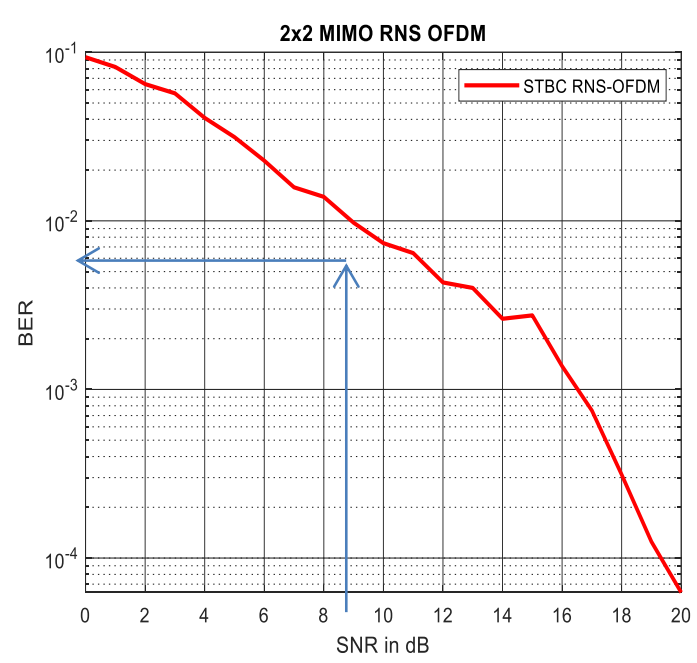

(b) Without mitigation scheme

Figure 10. MIMO-OFDM RNS system self-cancellation Scheme 


\subsection{MIMO-RNS-OFDM with "pulse shaping" scheme}

A raised cosine pulse shaping scheme added to the MIMO-RNS-OFDM system, and evaluated through the coming simulations.

\subsubsection{PAPR measurement}

Perform recurrent measurement to evaluate the PAPR of the communication system using RNS as coding and as error control scheme, with and without pulse shaping mitigation as seen in Figure 11. From Figure 11(a) and Figure 11(b), a reduction in PAPR with RNS coding and error control when using pulse shaping mitigation scheme in comparison to that without the mitigation scheme.

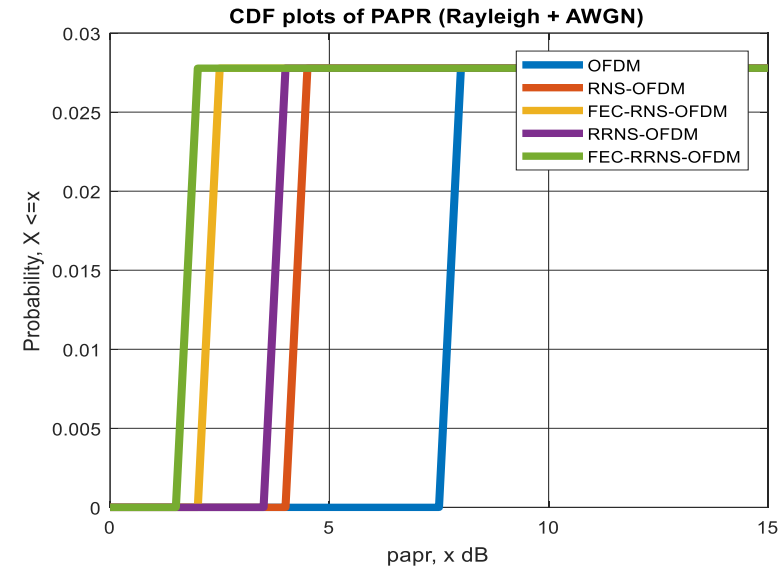

(a)

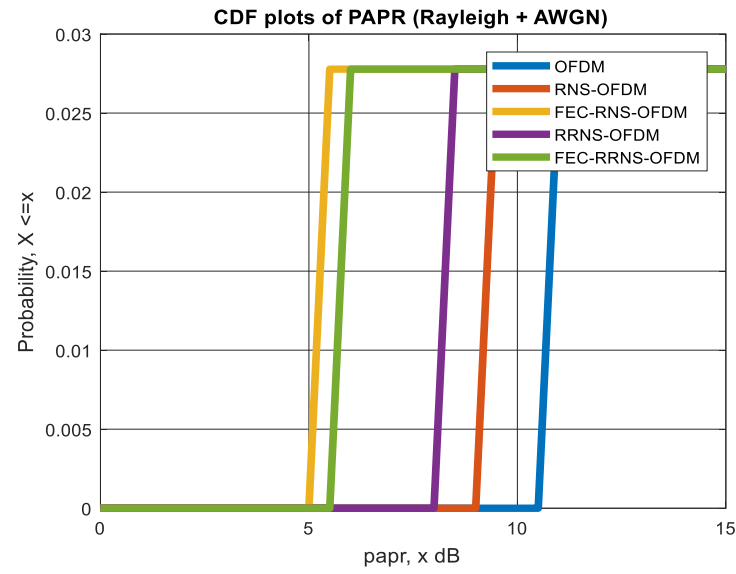

(b)

Figure 11. (a) With mitigation scheme, (b) Without mitigation scheme

\subsubsection{ICI and BER measurement}

Then measuring the ICI error and overall BER performance for the system with and without pulse shaping mitigation is measured, as seen in Figure 12 and Figure 13. From the above Figure 12 it is shown that using the mitigation scheme as in Figure 12(a) the ICI reduction using RNS coding is around $40 \mathrm{~dB}$ while without the mitigation scheme as seen in Figure 12(b) the reduction is only $30 \mathrm{~dB}$. The improved features seen in Figure 12(a) is attributed to the use of pulse shaping scheme as a mitigation technique in the communication system.

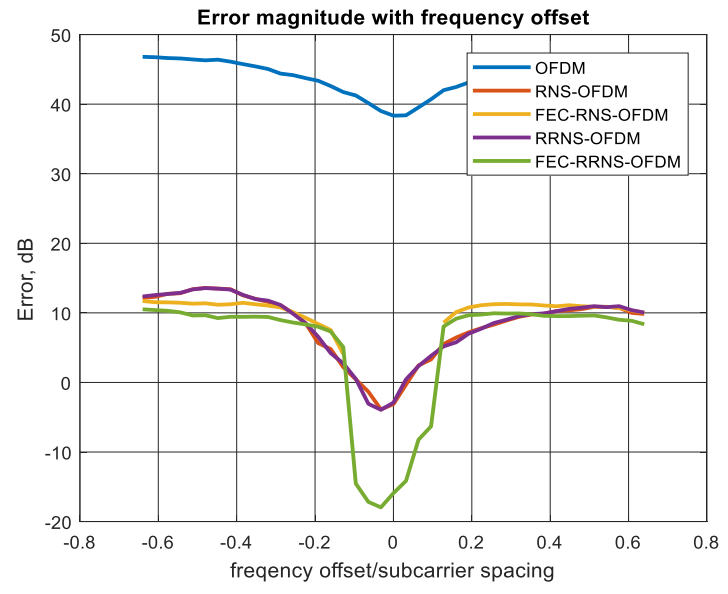

(a)

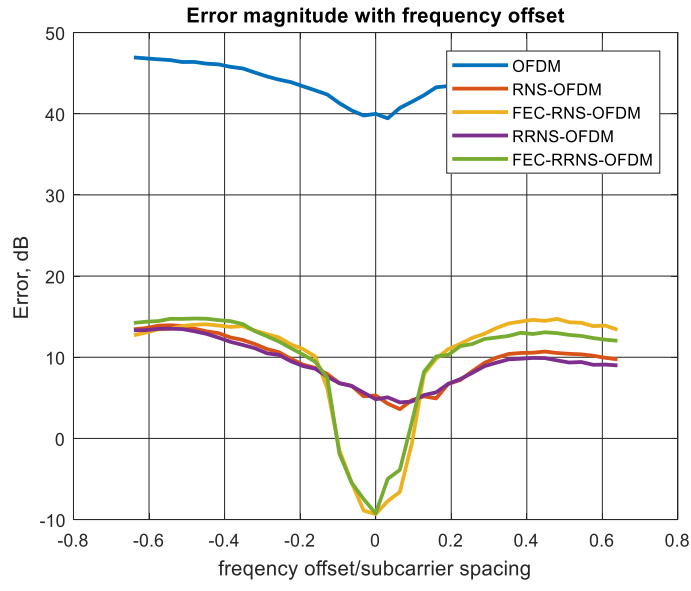

(b)

Figure 12. (a) ICI With mitigation scheme, (b) ICI Without mitigation scheme 
And from Figure 13 the BER for the system with and without mitigation scheme is analyzed; where it is shown that BER performance in Figure 13(a) is better than that seen in Figure 13(b). At a $\mathrm{SNR}=10 \mathrm{~dB}$, the BER for the RRNS communication system with mitigation scheme seen in Figure 13(a) is $10^{-4}$ while for the same system without mitigation scheme as seen in Figure 13(b) is $10^{-3}$. This result is coherent with that obtained in Figure 12 indicating the decrease of ICI when implementing mitigation scheme.

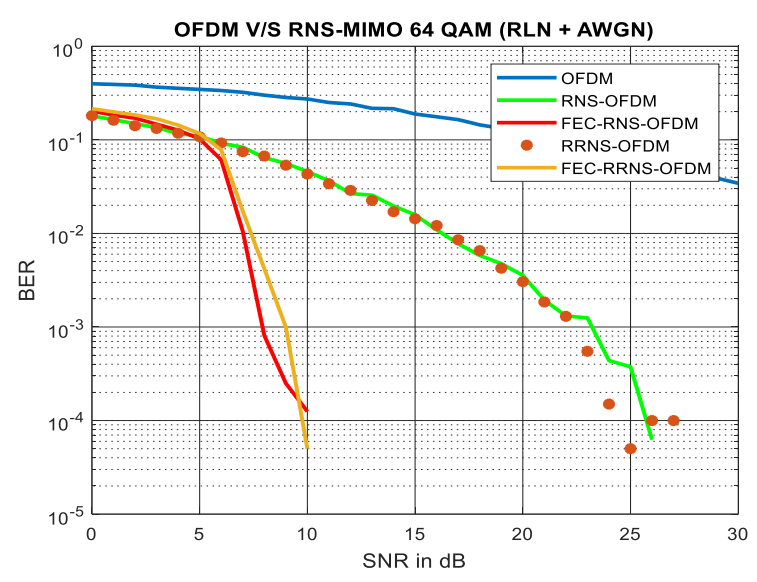

(a)

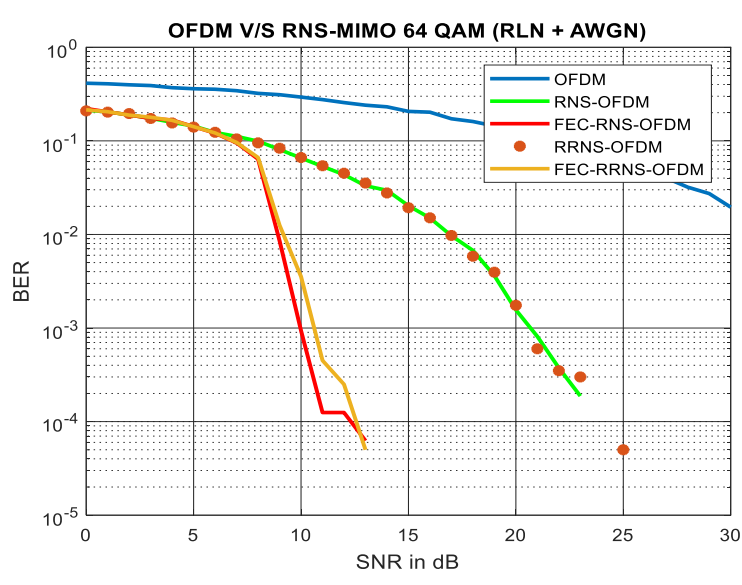

(b)

Figure 13. (a) BER With pulse shape mitigation, (b) BER Without pulse shape mitigation

\subsection{MIMO-RNS-OFDM vs. ICI reduction techniques}

In this subSection a comparisons of various ICI cancelation schemes that are implemented within the MIMO-RNS-OFDM system using 32-QAM over a Rayliegh fading channel, are studied and analyzed as seen in Figure 14, to determine the best choice of ICI mitigation techniques that is suitable of RNS coding scheme. The ICI and BER seen in Figure 14 improve when utilizing a mitigation technique with the proposed RNS coding scheme. Some mitigation techniques have better performance as compared to others, where the Raised cosine pulse shaping provide better performance compared to that when using the equalization scheme; through maintaining less ICI and better bandwidth efficiency.

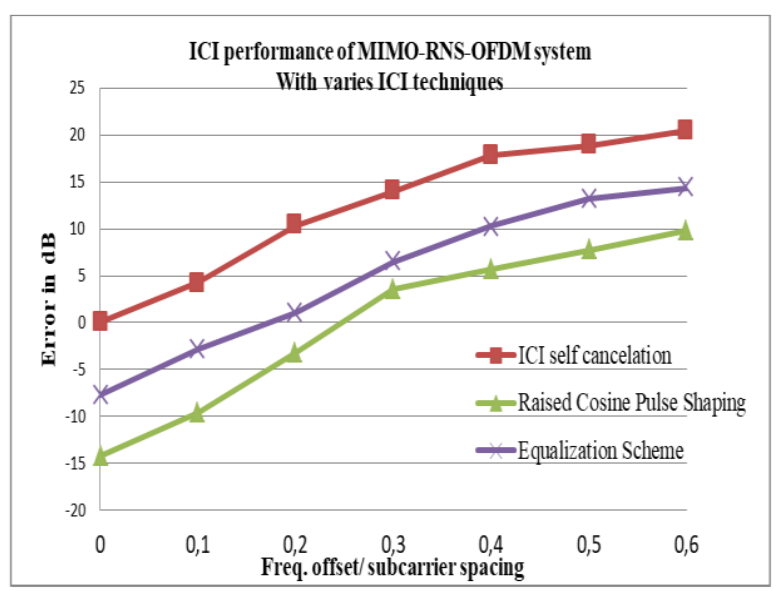

(a)

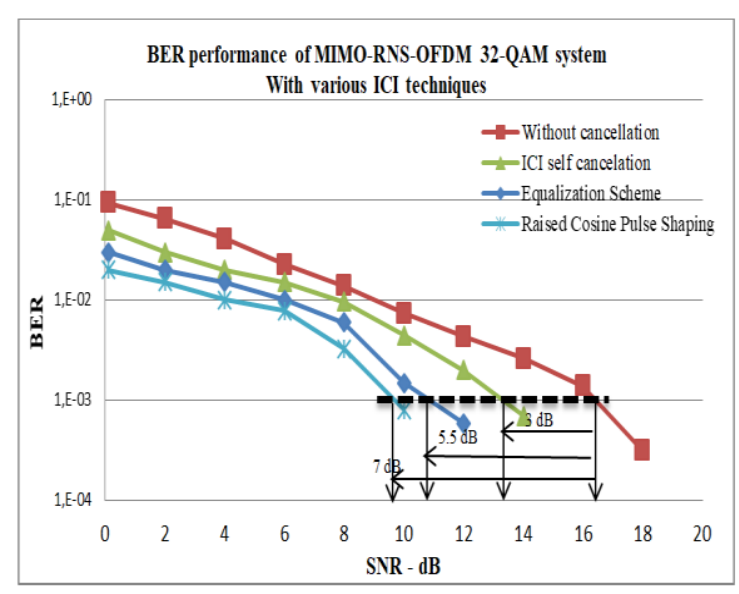

(b)

Figure 14. (a) ICI for MIMO-RNS-OFDM with varies mitigation schemes, (b) BER for MIMO-RNS-OFDM with varies mitigation schemes 


\section{CONCLUSION}

In this paper, a review for MIMO-OFDM system performance using ICI self-cancelation, pulse shaping, windowing mitigation techniques had been provided and discussed. An RNS coding insertion in MIMO-OFDM communication system has been proposed, and evaluated with respect to both CIR and BER performance. The usage of residue system had showed its advantage in improving the communication system features through decreasing the ICI and improving the BER performance.

The MIMO-OFDM with RNS coding scheme further enhanced through the insertion of ICI mitigation technique in the communication system, where the pulse shaping mitigation scheme had proven its enhanced performance with the residue system over the equalization scheme; through the recorded improvement in the BER, PAPR and ICI parameters, due to that equalization and self-cancellation techniques only reduce the ICI caused by fading/multipath distortion, while ICI generated due to frequency mismatch between transmitter and receiver, and the Doppler shift are covered through pulse shaping approach.

\section{REFERENCES}

[1] Biradar, R. "Study and Analysis of 2x2 MIMO Systems for Different Modulation Techniques Using MATLAB," July, 2015. Retrieved September 10, 2017, from http://www.techrepublic.com/resource-library/whitepapers/studyand-analysis-of-2x2-mimo-systems-for-different-modulation-techniques-using-matlab/

[2] Saunders S R, "Antennas and Propagation for Wireless Communication Systems," Wiley, 1999.

[3] H. Yang, "A Road to Future Broadband Wireless Access: Mimo-Ofdm Based Air Interface," Communications Magazine, IEEE, vol. 43, pp.53-60, 2005.

[4] Theory of Frequency Division Multiplexing: http://zone.ni.com/devzone/cda/ph/p/id/269

[5] Ghassan M. T. Abdalla "Orthogonal Frequency Division Multiplexing. Theory and Challenges" UofKEJ, Vol. 1 Issue 2 pp. 1-8, October, 2011.

[6] T. Wang, J. G. Proakis, and J. R. Zeidler, "Performance Degradation of OFDM Systems Due to Doppler Spreading," IEEE Transactions on Wireless Communications, vol. 5, pp. 1422-1432, June 2006.

[7] M. Roshanzadeh, A. Ghaffari and S. Saqaeeyan, "Using Residue Number Systems for Improving QoS and Error Detection \& Correction in Wireless Sensor Networks, Communication Software and Networks (ICCSN)," IEEE 3rd International Conference on Page: 1-5, May 2011.

[8] Pallab Maji, "Application of Residue Arithmetic in Communication and Signal Processing," Master of Science. National Institute of Technology, Rourkela, Orissa-769008, India, June 2011.

[9] Aingel James, Ameenudeen Pe, Jilu James, Minny George, "Multiple Error Correction Using Non-Binary Redundant Residue Number System", India Conference (INDICON) 2015, ISSN 2325-9418, 2015.

[10] Hari Krisna, and Kuo-Yu Lin, A coding Theory Approach to Error Control in Redundant Residue Number Systems- Part I: Theory and Single Error Correction, IEEE Transactions on Circuits and Systems II: Analog and Digital Signal Processing Vol 39 issue 1 pp 8-17 Jan 1992

[11] Salifu Abdul-Mumin1, Kazeem Alagbe Gbolagade, "An Improved Redundant Residue Number System Based Error Detection and Correction Scheme for the Moduli Set," Advances in Wireless Communications and Networks journal, 2(1): 11-14, 2016.

[12] Jasdeep Singh, Komal Arora, "Inter carrier interference removal in MIMO-OFDM system," IOSR Journal of Electronics and Communication Engineering (IOSR-JECE), e-ISSN: 2278-2834, p-ISSN: 2278-8735.Volume 9, Issue 2, Ver. VII (Mar - Apr. 2014), PP 87-91, 2014.

[13] H. M. Mourad, "Reducing ICI in OFDM Systems using a Proposed Pulse Shape,"Wireless Person. Communication, Vol. 40, pp. 41-48, 2006.

[14] V. Kumbasar and O. Kucur, "ICI Reduction in OFDM Systems by usingImproved Sinc Power Pulse," Digital Signal Processing, Vol.17, Issue 6, pp.997-1006, Nov. 2007.

[15] Mrityunjaya Hatagundi1, Ramakrishna Joshi "ICI Cancellation using Self Cancellation Method," International Journal of Advanced Research in Computer and Communication Engineering, ISO 3297:2007 Certified Vol.5, Issue 12, ISSN (Online) 2278-1021, December 2016.

[16] Yin-Ray Huang, Carrson C., "Frequency Domain Equalization for OFDM Systems with Insufficient Guard Interval using null subcarriers,"17th European Signal Processing Conference (EUSIPCO 2009) Glasgow, Scotland, August 24-28, 2009

[17] R.Kumar, S.Malarvizhi, "Time Domain Equalization Technique for Intercarrier Carrier Interference Suppression in OFDM System," Information Technology Journal, ANSINET, Vol 7(1), PP.149-154,1-jan-2008.

[18] M. Palanivelan, Sheila Anand "PAPR and ICI Reduction in OFDM Systems using Modified Raised Cosine Power Pulse Shape," European Journal of Scientific Research. Vol.72 No.4 (2012), pp. 618-627.

[19] Kusumasri Mallikanti, "Analysis of OFDM System by Using Pulse Shaping Filters for DSP Applications," SSRG International Journal of Electronics and Communication Engineering- (ICRTESTM-2017) - Special Issue- April 2017, ISSN: 2348 - 8549, 2017. 